\title{
Rapid emergence and antigenic diversification of the norovirus 2012 Sydney variant in Denmark, October to December, 2012
}

J Fonager (Fon@ssi.dk) ${ }^{1}$, L S Hindbæk ${ }^{1}$, T K Fischer ${ }^{1}$

1. Department of Microbiological Diagnostics and Virology, Statens Serum Institut, Copenhagen, Denmark

Citation style for this article:

Fonager J, Hindbæk LS, Fischer TK. Rapid emergence and antigenic diversification of the norovirus 2012 Sydney variant in Denmark, October to December, 2012. Euro Surveill. 2013;18(9):pii=20413. Available online: http://www.eurosurveillance.org/ViewArticle.aspx?Articleld=20413

Article submitted on 25 February 2013 / published on 28 February 2013

The norovirus (NoV) season in Denmark in late 2012 was characterised by an increase in the number of NoV infections caused mainly by the 2012 Sydney variant, but also by the 2009 New Orleans variant. Analysis of approximately $85 \%$ of the capsid gene from 10 Sydney 2012 and 9 New Orleans 2009 isolates showed rapid antigenic diversification of the Sydney 2012 variant shortly after its emergence. We also present new primers useful for transmission tracking.

Emergence of the norovirus 2012 Sydney variant in Denmark

In Denmark, we initially identified the norovirus (NoV) GGII.4 2012 Sydney variant in a geographically localised outbreak in November 2012 and simultaneously observed a rapid increase in the proportion of NoV infections caused by this variant. This emerging variant has been reported in two recent European studies $[1,2]$. In light of these, we re-examined our data and found earlier occurrences of the Sydney 2012 variant. We also used new primers, developed to amplify GGII.4 variants, which enabled characterisation of about $85 \%$ of the NoV capsid gene.

A subset of NoV-positive samples obtained from routine diagnostics and outbreaks were selected for genotyping from months in which most NoV cases with each variant were observed: 251 of 1,056 NoV-positive samples in 2011 and 213 of 670 NoV-positive samples in 2012. We analysed NoV polymerase and/or capsid

\section{FIGURE 1}

Norovirus types in Denmark $2011(\mathrm{n}=251)$ and $2012(\mathrm{n}=213)$

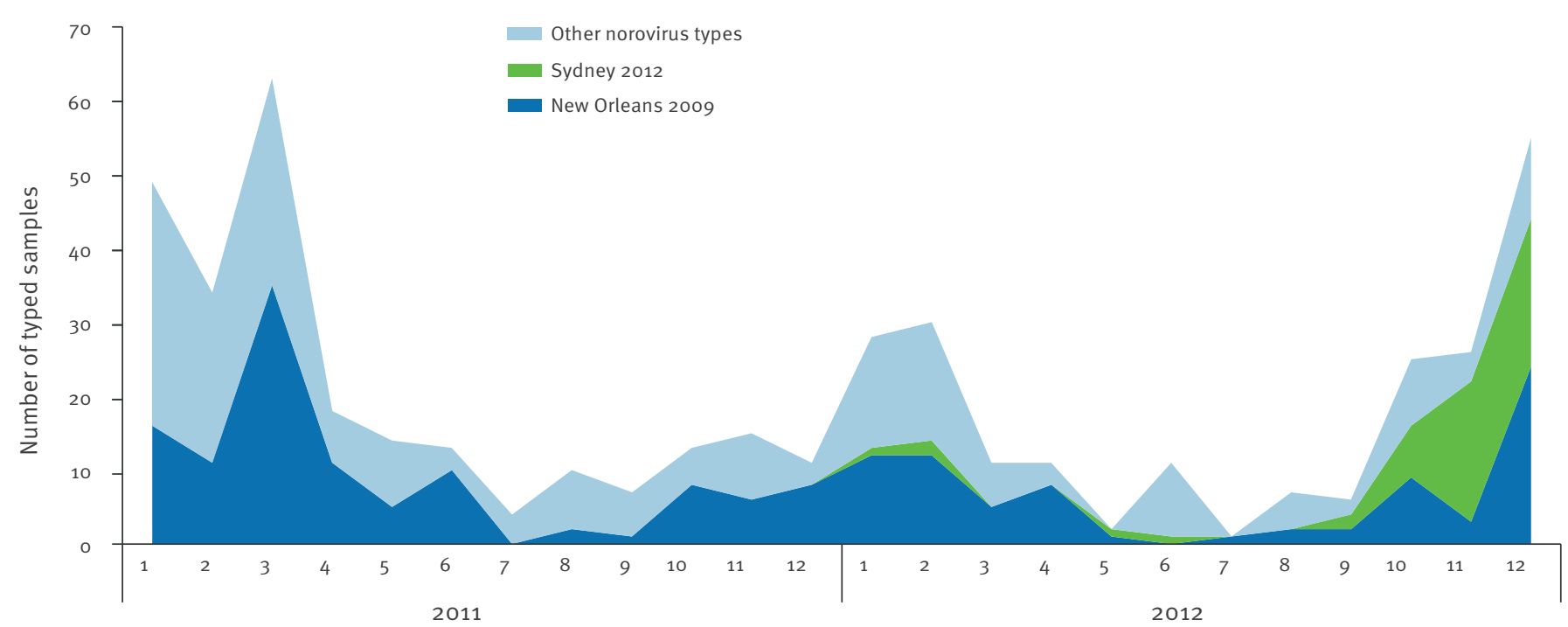


Primers used for RT-PCR amplification and sequencing of the norovirus capsid gene

\begin{tabular}{|l|c|c|c|c|c|}
\hline Primer name & Orientation & Used in & Sequence (5' to 3') & Positions & Size (bp) \\
\hline GGII.4 F1 & F & 1 PCR & GCACGTGGGAGGGCGATCG & 5043 to 5061 & - \\
\hline GGII.4 R1 & R & 1 PCR & GCCAATCCAGCAAAGAAAGCTCCAG & 6711 to 6735 & 1,692 \\
\hline GGII.4 F2 & F & 2 PCRs & CAGCCAACCTCGTCCCAGAGGTC & 5128 to 5150 & - \\
\hline GGII.4 R2 & R & 2 PCRs & CACGTCTACGCCCCGTYCCATTTCC & 6675 to 6699 & 1,571 \\
\hline GGII.4 F3(Seq) & F & Sequencing & CACCACTTAGGGCYAAYATGCTGG & 5635 to 5659 & - \\
\hline GGII.4 R3(Seq) & R & Sequencing & CCAGCATTRTTRGCCCTAAGTGGTG & 5635 to 5659 & - \\
\hline GGII.4 F4(Seq) & F & Sequencing & GATGTCACCCACATTGCAGGTTCTCG & 5649 to 5974 & - \\
\hline GGII.4 R4(Seq) & R & Sequencing & CGAGAACCTGCAATGTGGGTGACATC & 5649 to 5974 & - \\
\hline
\end{tabular}

bp: base pairs; F: forward; R: reverse; RT-PCR: reverse transcription polymerase chain reaction.

Positions are indicated relative to the GU445325.2 reference sequence.

sequences obtained by reverse transcription polymerase chain reaction (RT-PCR) using standard typing primers [3-8]. Genotypes were assigned to one of three categories (Figure 1), according to the capsid and/ or polymerase sequences. In cases where only polymerase gene sequences were available, exclusively top BLASTN hits (National Center for Biotechnology Information) against the 2012 Sydney variant reference sequence or, alternatively, genotype identification via the NoV typing tool [9] were used for variant designation. We found no evidence of recombination between any GGII polymerase and II.4 capsid genes among 132 double (both polymerase and capsid) genotyped samples from 2011 and 2012, and therefore assigned all non-Il.e polymerase gene sequences (2012 Sydney variant) to the category other NoV types (Figure 1).

The results show that the 2009 New Orleans variant was the single most dominant variant during 2011 and until April 2012, accounting for 150 of 331 (45\%) typed samples, whereas the 2012 Sydney variant was only sporadically detected in routine diagnostics and outbreak samples from January 2012 to October 2012, after which it rapidly emerged as the dominant variant in both surveillance and outbreak samples, accounting for 46 of 106 (43\%) typed samples from October 2012 to December 2012 (Figure 1). However, during this period, the New Orleans 2009 variant still accounted for 36 out of 106 (34\%) typed samples, making it too early to predict whether the Sydney 2012 variant will entirely replace the New Orleans variant.

Further characterisation of both variants was performed through phylogenetic analysis of nucleotide and the deduced amino acid sequences - using neighbor joining with Jukes-Cantor model of substitution (nucleotides) and the number of differences (amino acids) respectively, in MEGA5 [10] - of nearly complete capsid gene sequences obtained through RT-PCR with primers described in this report (Table).
In total, 10 Sydney 2012 variants (obtained from October to December 2012) and nine New Orleans 2009 variants (obtained from March 2011 to December 2012) were selected from the months with the highest number of cases observed for each variant for analysis of the capsid gene*. Included in this set were 12 samples from four outbreaks (Figure 2). All outbreaks occurred in a geographically localised area and the following number of samples were sent from each outbreak: outbreak 1 (18 samples from nine persons), outbreak 2 (seven samples from three persons), outbreak 3 (three samples from three persons) and outbreak 4 (eight samples from seven persons) The remaining samples of each variant were from our routine diagnostics and did not belong to any known outbreak. The primers were able to amplify the capsid region of both variants, which will make them a valuable tool in transmission tracking of both variants. For both variants, $100 \%$ identical sequences were observed within each of the four outbreaks (apart from outbreak 4, where two nucleotide differences were observed in one sequence), which indicates a probable point source of infection and also shows that sequencing of the capsid region using these primers is a reliable way of identifying a probable point source of infection caused by the 2012 Sydney variant.

\section{Antigenic diversification of norovirus variants}

In order to assess the functional divergence of the two variants, we translated the almost-complete capsid gene sequences $(n=19)$ into amino acids, and found five different branches for the Sydney 2012 variant and six different branches for the New Orleans 2009 variant (Figure 3). We also investigated the amino acid variations occurring in the predicted GII.4 blockage epitope sites [11] and found three distinct patterns of amino acid substitutions compared with the reference sequences for both variants (Figure 4 ). Although more amino acid substitutions occurred in the New Orleans 


\section{FIGURE 2}

Neighbor-joining (Jukes-Cantor) phylogenetic tree of approximately $85 \%$ of the complete capsid gene nucleotide sequences and the Sydney 2012 (JX459908) and New Orleans 2009 (GU445325.2) reference sequences

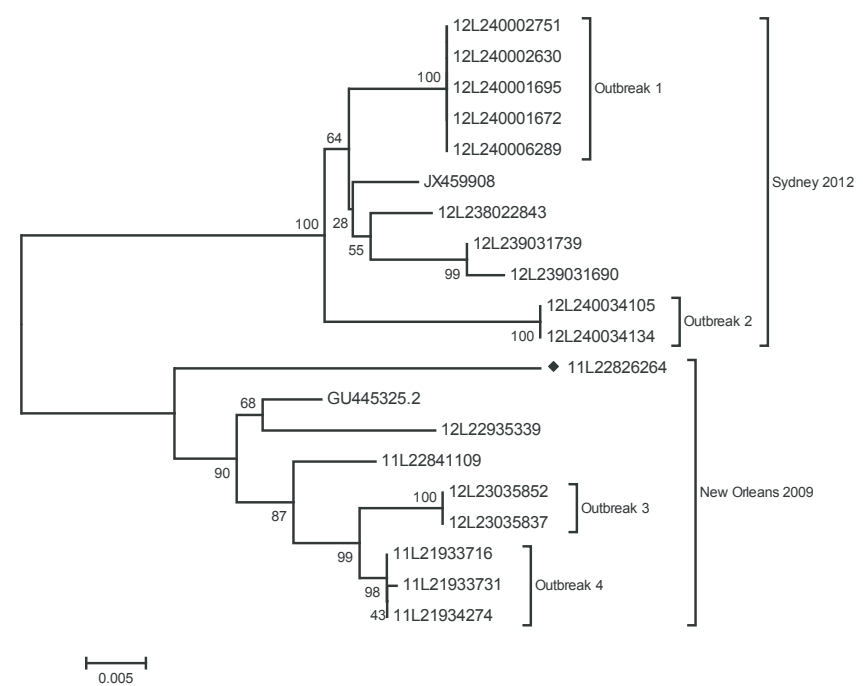

The following isolates were analysed (sample collection day/ month/year in parentheses): 11L21933716 (01/03/11),11L21933731 (18/03/11), 11L21934274 (21/03/11), 11L22826264 (16/12/11), 11 L 22841109 (23/12/11), 12L22935339 (20/01/12), 12L23035852 (24/02/12), 12L23035837 (24/02/12), 11L240026757 $(18 / 12 / 12)$ and 10 Sydney 2012 isolates: 12 L238022843 (15/10/12), 12L239031739 (17/11/12), 12L239031690 (19/11/12), $12 \mathrm{~L} 240001672$ (03/12/12), 12L240001695 (03/12/12), 12 L240002751 (04/12/12), 12 L240002630 (04/12/12), $12 \mathrm{~L} 240006289$ (05/12/12), $12 \mathrm{~L} 240034134$ (22/12/12), $12 \mathrm{~L} 240034105(23 / 12 / 12)$.

A very diverse New Orleans 2009 sequence is indicated by a black diamond.

variants (five substitutions) than in the Sydney 2012 variant (three substitutions), the time interval from the first to the last New Orleans 2009 variant was 647 days, compared with only 68 days for the Sydney 2012 variant, showing that the Sydney 2012 variant is displaying a potentially diverse antigenic repertoire shortly after its emergence in Denmark. Interestingly, one of the New Orleans 2009 sequences (obtained just 17 days before the first Sydney 2012 variant was identified in Denmark) was found to be very distinct in both the phylogenetic analysis and alone accounted for two of the five amino acid substitutions in the antigenic sites of all New Orleans variants analysed (Figure 4). This could indicate the presence of a strong selection pressure on the 2009 New Orleans variants for functional variation to avoid accumulated herd immunity, while the newly introduced 2012 Sydney variant can circulate and diversify much more freely in the population.

\section{Conclusion}

In summary, we have documented the rapid emergence of the Sydney 2012 variant as the dominant NoV type in Denmark. Although it is still too early to predict whether the Sydney 2012 variant will replace the New Orleans 2009 variant, our analyses of the capsid gene demonstrate that this variant has the potential for strain replacement as it is rapidly diversifying within the Danish population. In addition, we present and demonstrate the successful use of new primers, which can amplify approximately $85 \%$ of the capsid (and the hypervariable $\mathrm{P}_{2}$ ) region. The primers can therefore be used to perform a detailed comparison of sequences and thereby assist in transmission tracking of the new 2012 Sydney variant.

\section{FIGURE 3}

Neighbor joining (number of differences) phylogenetic analysis of translated capsid sequences

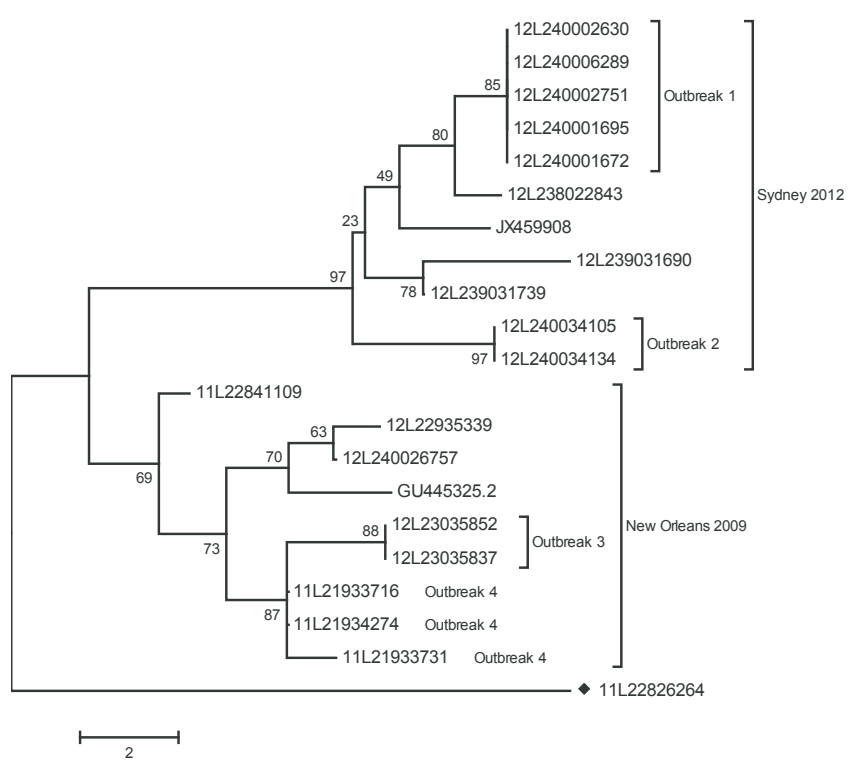

The following isolates were analysed (sample collection day/ month year in parentheses): 11L21933716 (01/03/11),11L21933731 (18/03/11), 11L21934274 (21/03/11), 11L22826264 (16/12/11), $11 \mathrm{~L} 22841109$ (23/12/11), 12L22935339 (20/01/12), 12L23035852 (24/02/12), 12L23035837 (24/02/12), 11L240026757 $(18 / 12 / 12)$ and 10 Sydney 2012 isolates: 12 L238022843 (15/10/12), 12L239031739 (17/11/12), 12L239031690 (19/11/12), 12L240001672 (03/12/12), 12L240001695 (03/12/12), $12 \mathrm{~L} 240002751$ (04/12/12), 12L240002630 (04/12/12), 12 L240006289 (05/12/12), 12 L240034134 (22/12/12), $12 \mathrm{~L} 240034105(23 / 12 / 12)$.

A very diverse New Orleans 2009 sequence is indicated by a black diamond. 


\section{FIGURE 4}

Variation in the predicted antigenic blockade epitope sites (A, D and E) for the New Orleans 2009 and Sydney 2012 variants (var 1-6)

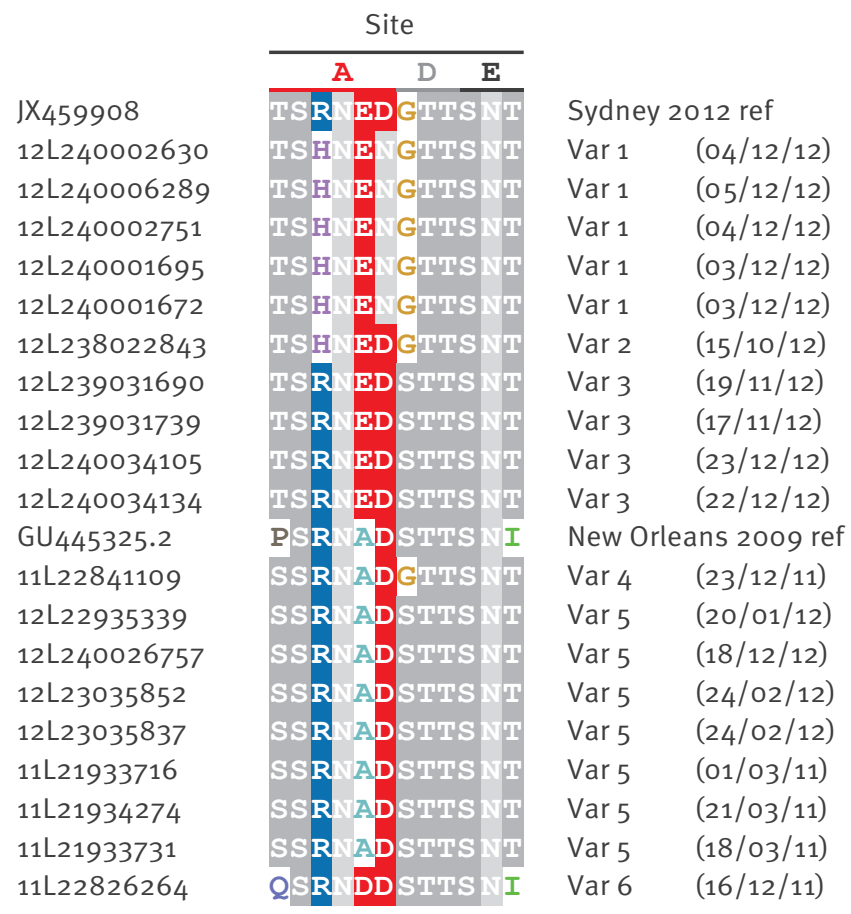

Observed epitope variants, sample collection dates (day/month/ year) and reference sequences are indicated on the right of the figure. Positions of the epitopes are indicated relative to the translated capsid (VP1) New Orleans reference sequence (GU445325.2): A site (left to right): 294, 296, 297, 298, 368, 372; D site (left to right): 393, 394, 395; E site (left to right): 407, 412, 413.

\section{${ }^{*}$ Addendum}

An addendum for this article was published on 7 March 2013, listing the GenBank accession numbers for the capsid genes used in the phylogenetic analysis.

\section{Acknowledgements}

We thank the Danish Veterinary and Food administration and the Department of Epidemiology, Statens Serum Institut for obtaining and providing samples from outbreaks which we have used in the phylogenetic analysis in this study. In particular we would like to thank Morten Lisby, Annette Villif, Karina Hindsdal, Hanne M Holt, Anne R Larsen, Sofie $\mathrm{G}$ Lassen and Lieke v Alphen. Furthermore, we would like to thank all general practitioners and Clinical Microbiological departments in Denmark, who provided samples for norovirus analysis. We greatly appreciate and value the high technical skills of the laboratory technicians Jesper Rønn and Camilla Dalgaard.
Authors' contributions

J Fonager: Conceived the idea for the study and performed the phylogenetic analysis and antigenic characterisations and drafted the first version of the paper, made revisions, and approved the final version of the paper. TK Fischer: Designed the project together with J Fonager, provided constructive comments and revised the first draft of the paper critically and approved the final version of the paper. LS Hindbæk: Contributed considerably with the laboratory analyses and approved the final version of the paper.

\section{References}

1. van Beek J, Ambert-Balay K, Botteldoorn N, Eden J, Fonager J, Hewitt I, et al. Indications for worldwide increased norovirus activity associated with emergence of a new variant of genotype II.4, late 2012. Euro Surveill. 2013;18(1): pii=20345. Available from: http://www.eurosurveillance.org/ViewArticle. aspx?Articleld $=20345$

2. Bennett S, MacLean A, Miller RS, Aitken C, Gunson RN. Increased norovirus activity in Scotland in 2012 is associated with the emergence of a new norovirus Gll.4 variant. Euro Surveill. 2013;18(2):pii=20349. Available from: http://www. eurosurveillance.org/ViewArticle.aspx?Articleld=20349

3. Gallimore Cl, Cheesbrough JS, Lamden K, Bingham C, Gray JJ. Multiple norovirus genotypes characterised from an oysterassociated outbreak of gastroenteritis. Int J Food Microbiol. 2005;103(3):323-30.

http://dx.doi.org/10.1016/j.ijfoodmicro.2005.02.003 PMid:15967530

4. Hoebe CJ, Vennema H, de Roda Husman AM, van Duynhoven YT. Norovirus outbreak among primary schoolchildren who had played in a recreational water fountain. J Infect Dis. 2004;189(4):699-705.

http://dx.doi.org/10.1086/381534

PMid:14767824

5. Kageyama T, Kojima S, Shinohara M, Uchida K, Fukushi S, Hoshino FB, et al. Broadly reactive and highly sensitive assay for Norwalk-like viruses based on real-time quantitative reverse transcription-PCR. J Clin Microbiol. 2003;41(4):1548-57. http://dx.doi.org/10.1128/JCM.41.4.1548-1557.2003 PMid:12682144 PMCid:153860

6. Kojima S, Kageyama T, Fukushi S, Hoshino FB, Shinohara M, Uchida K, et al. Genogroup-specific PCR primers for detection of Norwalk-like viruses. J Virol Methods. 2002;100(1-2):107-14.

7. Le Guyader F, Neill FH, Estes MK, Monroe SS, Ando T, Atmar RL. Detection and analysis of a small round-structured virus strain in oysters implicated in an outbreak of acute gastroenteritis. Appl Environ Microbiol. 1996;62(11):4268-72.

8. Vennema H, de Bruin E, Koopmans M. Rational optimization of generic primers used for Norwalk-like virus detection by reverse transcriptase polymerase chain reaction. J Clin Virol. 2002;25(2):233-5. http://dx.doi.org/10.1016/S1386-6532(02)00126-9

9. Kroneman A, Vennema H, Deforche K, v d Avoort H, Peñaranda $\mathrm{S}$, Oberste MS, et al. An automated genotyping tool for enteroviruses and noroviruses. J Clin Virol. 2011;51(2):121-5. http://dx.doi.org/10.1016/j.jcv.2011.03.006 PMid:21514213

10. Tamura K, Peterson D, Peterson N, Stecher G, Nei M, Kumar S. MEGA5: molecular evolutionary genetics analysis using maximum likelihood, evolutionary distance, and maximum parsimony methods. Mol Biol Evol. 2011;28(10):2731-9. http://dx.doi.org/10.1093/molbev/msr121 PMid:21546353 PMCid:3203626

11. Debbink K, Lindesmith LC, Donaldson EF, Baric RS. Norovirus immunity and the great escape. PLoS Pathog. 2012;8(10):e1002921.

\section{Conflict of interest}

None declared. 